\title{
The influence of pregnancy-induced hypertension syndrome on the metabolism of newborns
}

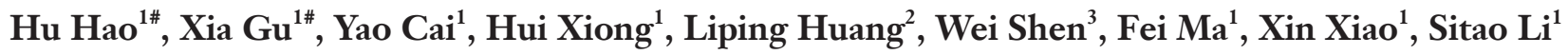 \\ ${ }^{1}$ Department of Neonatology, The Sixth Affiliated Hospital of Sun Yat-sen University, Guangzhou, China; ${ }^{2}$ Department of Obstetrics and \\ Gynecology, Southern Medical University, Nanfang Hospital, Guangzhou, China; ${ }^{3}$ Department of Neonatology, Southern Medical University, \\ Nanfang Hospital, Guangzhou, China \\ Contributions: (I) Conception and design: H Hao, S Li; (II) Administrative support: F Ma, X Xiao; (III) Provision of study materials or patients: X Gu, \\ Y Cai, H Xiong; (IV) Collection and assembly of data: L Huang, W Shen; (V) Data analysis and interpretation: X Gu; (VI) Manuscript writing: All \\ authors; (VII) Final approval of manuscript: All authors. \\ \#These authors contributed equally to this work. \\ Correspondence to: Professor Xiao Xin; Li Sitao. Department of Pediatrics, The Sixth Affiliated Hospital of Sun Yat-Sen University, 26 Yuancun \\ Erheng Road, Guangzhou 510655, China. Email: xiaoxin2@mail.sysu.edu.cn; lisit@mail.sysu.edu.cn.
}

Background: Pregnancy-induced hypertension (PIH) is associated with an increased number of neonatal complications, but its impact on neonatal metabolism remains unclear. This study aimed to investigate the differential metabolomics of infants born to mothers with and without PIH.

Methods: Blood samples of a total of 115 infants born to mothers with $(n=56)$ and without (n=59) PIH were collected and assigned to two groups, respectively, from the neonatal department of Sixth Affiliated Hospital of Sun Yat-Sen University. A tandem mass spectrometer was used to generate metabolic profiling of amino acid, free carnitine, and acyl-carnitines. The resulting data were analyzed using orthogonal partial least squares discriminant analysis based on the difference between infants born to mothers with or without PIH.

Results: A significant relationship was observed between the two groups (with and without PIH) in the metabolic fingerprint. According to the pattern recognition analysis combined with variance importance, 25 metabolites with high importance were found. The top ten substances were selected for analysis. Compared with infants born to mothers without PIH, glycine levels increased, and C14DC, C22, C4DC, C5:1, C6DC, C5-OH, proline, C14-OH, and C20 decreased in infants born to mothers with PIH.

Conclusions: Using liquid chromatography (LC)-MS/MS metabolomics, a significant relationship was detected between neonatal metabolism and maternal hypertension. It is important to correct the subsequent infantile metabolic disorder by balancing the biomarker metabolites and suppling adequate nutrition to improve the health and growth of newborns of PIH mothers.

Keywords: Pregnancy-induced hypertension (PIH); neonatal metabolism; metabolomics; tandem mass spectrometer; nutrition

Submitted Jul 31, 2020. Accepted for publication Dec 23, 2020.

doi: $10.21037 / \mathrm{tp}-20-211$

View this article at: http://dx.doi.org/10.21037/tp-20-211

\section{Introduction}

Pregnancy-induced hypertension (PIH) is characterized by the de novo development of blood pressure above the normal level that arises $\geq 20$ weeks of gestation, accompanied by physiological and pathological changes, such as convulsion, coma, and heart and kidney disease/ failure in severe cases (1-5). The incidence rate is about $5-12 \%$, and the mortality rate is as high as $11-16 \%$ (6). In recent years, the incidence of PIH has grown, in parallel with an increase in mothers of advanced maternal age, 
increased incidence of female obesity and rheumatoid immune diseases, the demand for assisted reproductive technology, and other high-risk factors (7). At present, this disease's pathogenesis is not clearly understood, which brings great challenges for its treatment and prevention (8). Various maternal and neonatal complications can be caused by PIH and/or chronic hypertension, including acute liver and kidney failure, placental abruption, hemorrhage, growth restriction, small size for gestational age, asphyxia, or hypoxic ischemic encephalopathy, among others $(9,10)$.

Since the introduction of tandem mass spectrometry to newborn screening in 1990, its high sensitivity, high specificity, and rapid detection have propelled its development to become the ideal analytic technology for newborn screening in the past 20 years $(11,12)$. There are many reports in the literature that recommend using this technique to detect metabolites such as amino acids, organic acids, fatty acids, and steroid hormones in samples such as urine, blood, amniotic fluid, and cells to detect genetic metabolic diseases $(13,14)$. Metabolomics, as a discipline that was developed in the mid-1990s, is an emerging omics approach following transcriptomics and proteomics, and is an important part of systems biology (15-17). It can be used not only for disease diagnosis and prognosis assessment, but also for a multi-parameter judgment index of the body's metabolic homeostasis (18).

To our knowledge, the findings of metabolomics of PIH have been reasonably consistent between studies, with lipids and carnitines among the most commonly highlighted metabolites. These results have supported the existence of a PIH metabolome that is predominantly characterized by dysregulated fatty acid metabolism, which can be measured in a variety of biological samples, including blood, urine, placental tissue, and breast milk (19). Some studies have shown that PIH impacted the metabolism of bilirubin and lipid in newborns.

However, to date, research on the effects of PIH on newborns' postnatal metabolism has been scarce. Therefore, the mass spectrometry (MS)/MS-based metabolomics platform in this study used the mothers of newborns with normal pregnancies as controls, and the metabolites were analyzed to study the differences in blood metabolites among them in order to evaluate the impact of maternal hypertension during pregnancy on the blood metabolic status of the newborn and to provide reasonable nutritional supplement recommendations for further correction of metabolic disorders.

We present the following article in accordance with the strengthening the reporting of observational studies in epidemiology (STROBE) reporting checklist (available at http://dx.doi.org/10.21037/tp-20-211).

\section{Methods}

The study was conducted in accordance with the Declaration of Helsinki (as revised in 2013) and was approved by Ethics Committee of the Sixth Affiliated Hospital of Sun Yat-sen University (2017ZSLYEC-105). The blood samples of newborns were obtained after their parents had provided written informed consent.

\section{Data collection}

The participant inclusion criteria were as follows: (I) there were no complications during pregnancy, except PIH for mothers with $\mathrm{PIH}$, and no complications during pregnancy for normal mothers; (II) no history of unexplained death or premature death in the family; (III) no previous maternal dietary carnitine deficiency or genetic causes, and no history of alcohol, drug, tobacco, or substance abuse;

The exclusion criteria were as follows: (I) Refusal by family members for the newborn to undergo metabolism testing; (II) Severe complications (such as neonatal sepsis, congenital genetic metabolic disease, neonatal necrotizing enterocolitis, and severe metabolic disorder, among others) or death; (III) Congenital malformations and genetic metabolic diseases; (IV) Other perinatal complications, such as gestational diabetes mellitus, intrahepatic cholestasis during pregnancy, heart disease, hepatitis b virus infection, and hyperthyroidism or hypothyroidism, among others.

A total of 56 patients with PIH (average age 23-35) who were admitted between September 2010 and August 2012 to the Sixth Affiliated Hospital of Sun Yat-sen University were selected as the case group, and 59 normal pregnant women who were hospitalized or delivered in the hospital during the same period were selected as the control group. The heel Blood samples from the infants' heels were collected the 3 days after birth and 30 minutes after feeding.

\section{Diagnostic criteria}

We employed PIH's diagnostic criteria as listed in the 9th edition of 2019 Obstetrics and Gynecology: Hypertension during pregnancy refers to hypertension $(\geq 140 / 90 \mathrm{mmHg})$ on two occasions, $\geq 4$ hours apart, after 20 weeks of gestation. 


\section{Samples collection}

After the parents had understood and signed the informed consent to screen neonatal genetic and metabolic diseases, the newborn blood samples were collected. After disinfection, heel blood was collected using a blood collection needle on the inside or outside of the heel near the $1 / 3$ of the heel. Blood droplets naturally penetrated the filter paper. Three blood spots were continuously collected, each blood spot diameter was $>8 \mathrm{~mm}$, and the front and back sides of the filter paper were fully infiltrated to ensure that the blood concentration was uniform and that there was no blood ring. After the blood collection, the blood tablets were placed in a cool and clean place for natural drying, while avoiding ultraviolet rays, sunlight, and external pollution. After passing inspection, the dried blood tablets were placed in sealed bags and stored in a $2-8^{\circ} \mathrm{C}$ refrigerator.

\section{Test methods}

The blood tandem mass spectrometer was a Waters ultraperformance liquid chromatography MS (I-class) tandem (Xevo-TQD, Waters Corp., Milford, MA, USA). It used non-derivatized succinylacetone and various amino acid and carnitine assay kits. After punching, an extraction solution containing $100 \mu \mathrm{L}$ of internal standard (8: 2 methanol: ultrapure water) was added, it was placed in a microplate shaker at $700 \mathrm{rpm}$ and $45^{\circ} \mathrm{C}$ for 45 minutes, and then centrifuged in a centrifuge at 4,000 rpm for 5 minutes. A $75 \mu \mathrm{L}$ sample of the supernatant was placed in a 96-well heatresistant $\mathrm{V}$ bottom plate, and the aluminum film was used for testing.

\section{Data analysis}

Qualitative analysis was performed according to the $\mathrm{m} / \mathrm{z}$ of the mass spectrum peaks. The concentration values of amino acids and fatty acids of the 115 samples were automatically calculated by quantitative analysis software, and analyzed by SPSS 23.0 statistical software (IBM Corp., Armonk, NY, USA). We used the Kolmogorov-Smirnov and Shapiro-Wilk tests to test the normality of the data. Accord with normal distribution and variance of the quantitative data with mean $\pm \mathrm{SD}\left(\bar{x}_{ \pm} \mathrm{SD}\right)$ was described, and inter-group comparison was performed by $t$-test. The quantitative data not conforming to normal distribution were described by median (M) (P25, P75), and the rank-sum test was used for comparison between groups. The qualitative data were described by frequency, and the chi-square $\left(\chi^{2}\right)$ test was used for comparison between groups. Orthogonal partial least squares discriminant analysis (OPLS-DA) was performed using the SIMCA14.0 data processing software. The OPLSDA analyzed the blood metabolism of the two groups of newborns and looked for key differential metabolites. Two key indicators for evaluating the matching degree of the OPLS-DA model $\left(\mathrm{R}^{2} \mathrm{X}\right.$ represented the interpretation rate of the $\mathrm{X}$ matrix; $\mathrm{R}^{2} \mathrm{Y}$ represented the interpretation rate of the $\mathrm{Y}$ matrix, both of which reflected the stability of the model, each of the two were $>0.5$, indicating that the model matches well; the closer to 1 , the higher the matching degree of the model). According to the OPLSDA model, the more the substances at both ends were biased, the greater their covariance and correlation was, and the blood metabolites with variable important plots (VIPs) weighted $>1$ were screened out from them, and the scatters of different individual substances were plotted. Differences were considered to be significant when $\mathrm{P}<0.01$.

\section{Results}

\section{Basic clinical data}

The maternal age, weight, height, prenatal weight with and without PIH, the gestational age, gender, birth weight, length, and circumference of the above participants were recorded respectively, as shown in Table 1 and Table 2.

There were no significant differences between mothers with or without $\mathrm{PIH}$ regarding age and height $(\mathrm{P}>0.05)$. However, the weight and body mass index (BMI) of mothers between the two groups were significantly different $(\mathrm{P}<0.001)$. The $\mathrm{PIH}$ women were more likely to be overweight. Overweight and obese women are at higher risk of developing PIH.

There were no significant differences between the infants born to mothers with and without PIH in terms of gender and head circumference $(\mathrm{P}>0.05)$. However, the gestational age, birth weight, and body length between the two groups were significantly different $(\mathrm{P}<0.001)$. The average gestational age, weight, and body length of newborns of mothers with PIH were lower than those of newborns of mothers with normal pregnancies.

\section{Results of blood metabolism analysis of neonatal group with pregnancy-induced bypertension and neonatal group with normal pregnancy}

In order to eliminate the effects of non-essential factors, 
Table 1 Comparison of the basic clinical data of pregnant mothers in the two groups

\begin{tabular}{lcccc}
\hline Variable & PIH $(\bar{x} \pm \mathrm{SD})$ & Normal $(\bar{x} \pm \mathrm{SD})$ & $\mathrm{t}$-value & $\mathrm{P}$ value \\
\hline Age (year) & $28.8 \pm 5.1$ & $27.2 \pm 4.3$ & -1.78 & 0.079 \\
Weight $(\mathrm{kg})$ & $72.5 \pm 8.8$ & $65.6 \pm 8.0$ & -3.58 & $<0.001$ \\
Height $(\mathrm{cm})$ & $159.7 \pm 4.4$ & $159.9 \pm 4.5$ & 0.25 & 0.803 \\
BMl $\left(\mathrm{kg} / \mathrm{m}^{2}\right)$ & $28.4 \pm 0.5$ & $25.6 \pm 0.4$ & -4.65 & $<0.001$ \\
\hline
\end{tabular}

$\mathrm{PIH}$, pregnancy-induced hypertension; BMI, body mass index; SD, standard deviation.

Table 2 Comparison of basic clinical data of neonatal in the two groups

\begin{tabular}{|c|c|c|c|c|}
\hline Variable & $\mathrm{PIH}(\bar{x} \pm \mathrm{SD})$ & Normal $(x \pm \mathrm{SD})$ & $\chi^{2} / t$-value & $P$ value \\
\hline Gender (male) & $50 \%$ & $84 \%$ & 0.207 & 0.711 \\
\hline Weight (g) & $2,564.8 \pm 630.8$ & $3,220.3 \pm 275.3$ & 7.156 & $<0.001$ \\
\hline Height (cm) & $47.1 \pm 3.0$ & $49.6 \pm 1.4$ & 4.053 & $<0.001$ \\
\hline
\end{tabular}

$\mathrm{PIH}$, pregnancy-induced hypertension; SD, standard deviation.

complete the maximum separation between the two groups, and find the differential metabolites with larger weights, we used the supervised OPLS-DA method to remove information that was irrelevant to the sample grouping. We analyzed the mothers with PIH full spectrum of neonatal blood metabolism compared to the normal pregnancy mothers. The OPLS-DA score chart of the full spectrum of blood metabolism data is shown in Figure 1. The model contained two principal components, $\mathrm{R}^{2} \mathrm{Xcum}$ $=0.508$ and $\mathrm{R}^{2} \mathrm{Ycum}=0.805$, indicating that the model had a better interpretation ability. The prediction rate was high, which supported the model's reliability, and there was no overfitting phenomenon. It can be seen from Figure 1 that the PIH mother's pregnancy neonatal group and the mother's normal-pregnancy newborn group were each distributed in two regions, indicating that there were significant differences in blood metabolome between the two groups.

\section{Analysis of differential substances in mothers with pregnancy-induced hypertension and mothers with normal pregnancy}

The variable importance score chart was the most commonly used method to evaluate the contribution of variables in the supervised analysis method using the
OPLS-DA model. Screening for substances with significant differences was based on VIPs $>1.0$. After analysis by the OPLS-DA model, we identified myristyl carnitine (C14DC) and docosa carnitine (C22), succinyl carnitine (C4DC), glycine (Gly), prenyl carnitine (C5: 1), adipoyl carnitine (C6DC), 3-hydroxyisovaleryl carnitine (C5-OH), proline (Pro), 3-hydroxymyristoyl carnitine (C14-OH), eicosyl carnitine (C20), ornithine (Orn), myristyl dienoyl carnitine (C14:2), cycladienoyl carnitine (C10: 2), 3-hydroxypalmitoyl carnitine (C16: 1-OH), cyclinoyl carnitine (C10:1), threonine (Thr), tryptophan (Trp), leucine (Leu), asparagine (Asn), hexacosyl carnitine (C26), glutamic acid (Glu), octadecadienoyl carnitine (C18: 2), hexanoyl carnitine (C6), malonyl carnitine (C3DC), and valine (Val); a total of 25 different metabolites with weights $>1$ (Figure 2). The differential substances ranked in the top 10 in weight were selected for analysis. The levels of these 10 amino acids and/or acyl carnitine are shown in Table 3.

\section{Scatter plot of differential metabolites}

Metabolomics analysis was performed on the PIH mothers and the normal pregnancy mothers, and a total of 25 differential metabolites were obtained. From the scatter plot analysis (Figure 3), it could be seen that compared with the normal pregnancy mothers, Gly showed an increasing 


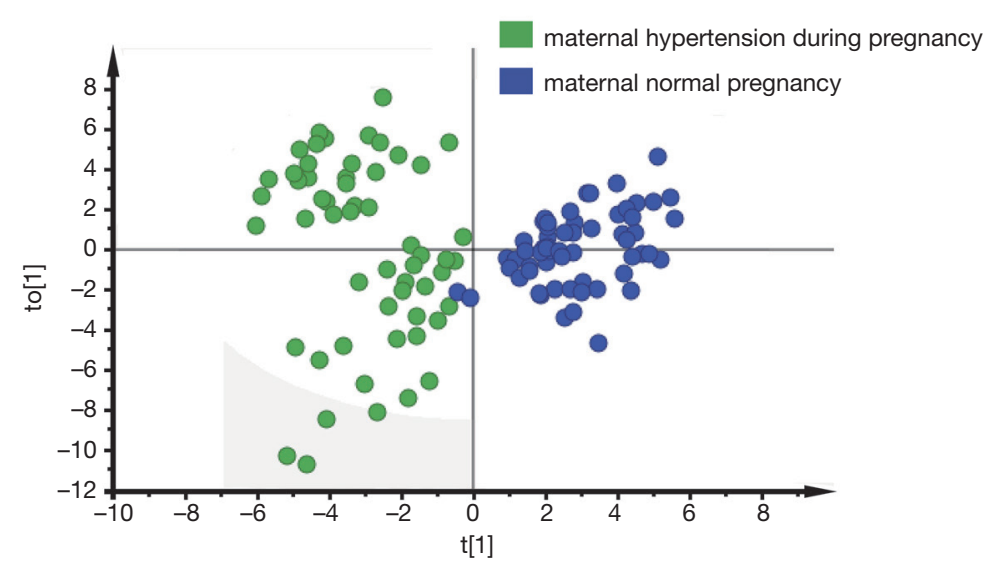

Note: the abscissa t[1] represents the predicted component, showing the difference between the groups; The vertical coordinate to[1] represents the orthogonal component and represents the degree of intra-group dispersion. The green dots indicate 56 maternal hypertension cases during pregnancy, and the blue dots indicate 59 cases of normal maternal pregnancy.

Figure 1 The OPLS-DA score plot of the neonatal group with maternal PIH and with normal pregnancy. OPLS-DA, orthogonal partial least squares discriminant analysis; $\mathrm{PIH}$, pregnancy-induced hypertension.

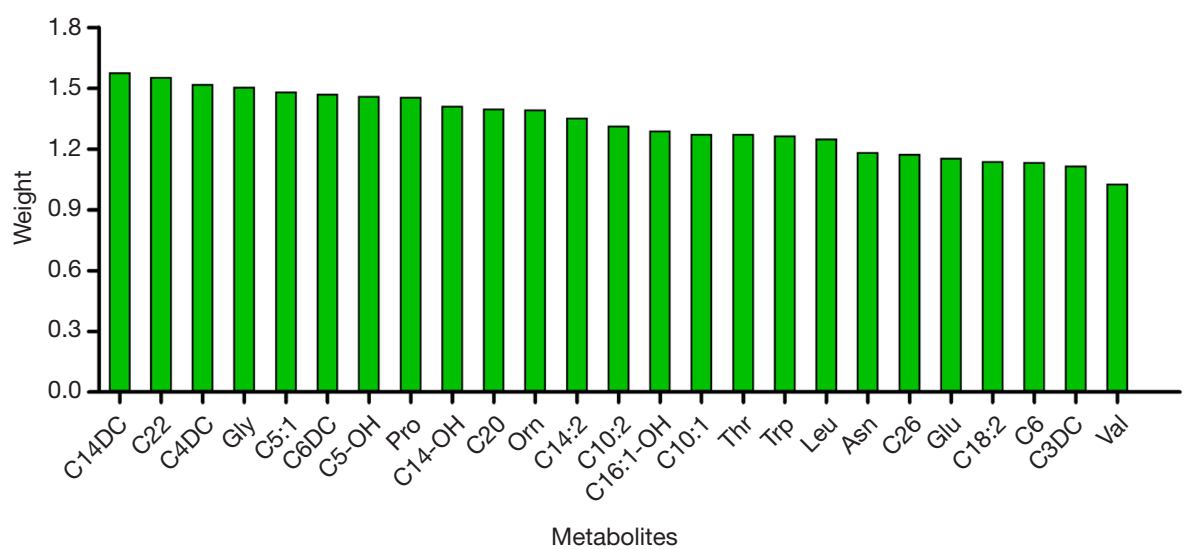

Figure 2 The variable important plot (VIP >1).

trend; C14DC, C22, C4DC, C5:1, C6DC, C5-OH, Pro, $\mathrm{C} 14-\mathrm{OH}$, and $\mathrm{C} 20$ showed a decreasing trend in the maternal PIH neonatal group.

Our research cohort included 59 cases of normal pregnancy, 11 cases of gestational hypertension, 18 cases of mild preeclampsia, 26 cases of severe preeclampsia, and 1 case of eclampsia. To understand the influence of PIH's severity on the newborn, we compared the different groups (as shown in Figure 4), but because of too small a number of eclampsia cases, here did not include it in the comparison.

As shown in Figure 4, the changes of blood metabolites in neonates between different degrees of $\mathrm{PIH}$ with normal pregnancy were statistically significant $(\mathrm{P}<0.001)$, while no statistical difference was detected between different degrees of $\mathrm{PIH}(\mathrm{P}>0.05)$.

According to the scatter plot, the level of Gly tended to increase with the increase of PIH degree, while the levels of Pro, C4DC, C14-OH, C14DC, and C20 tended to decrease. There was a trend of change but no statistical difference. Considering this study involved a small number and imbalance of samples, enlargement of the sample size in future research will help to verify these findings. 
Table 3 Levels of different substances in blood amino acid and fatty acid

\begin{tabular}{|c|c|c|c|c|}
\hline Material $(\mu \mathrm{mol} / \mathrm{L})$ & $\mathrm{PIH}(\mathrm{x} \pm \mathrm{SD}) / \mathrm{M}\left(\mathrm{P}_{25}, \mathrm{P}_{75}\right)$ & Normal $(x \pm S D) / M\left(P_{25}, P_{75}\right)$ & $\mathrm{t} / \mathrm{Z}$-value & $P$ value \\
\hline C14DC ${ }^{b}$ & $0.01(0,0.05)$ & $0.06(0.05,0.07)$ & 6.25 & $<0.001$ \\
\hline $\mathrm{C} 22^{\mathrm{b}}$ & $0.02(0.01,0.04)$ & $0.06(0.04,0.07)$ & 6.62 & $<0.001$ \\
\hline$C 4 D C^{a}$ & $0.18 \pm 0.09$ & $0.32 \pm 0.12$ & 7.46 & $<0.001$ \\
\hline Gly $^{a}$ & $274.43 \pm 122.75$ & $163.20 \pm 35.49$ & -6.53 & $<0.001$ \\
\hline $\mathrm{C}^{2} \mathrm{DC} \mathrm{b}^{\mathrm{b}}$ & $0.02(0.01,0.38)$ & $0.38(0.33,0.50)$ & 5.93 & $<0.001$ \\
\hline $\mathrm{C} 5-\mathrm{OH}^{\mathrm{a}}$ & $0.13 \pm 0.06$ & $0.20 \pm 0.05$ & 6.63 & $<0.001$ \\
\hline Pro $^{b}$ & $181.32(100.33,619.23)$ & $628.49(531.11,792.38)$ & 5.83 & $<0.001$ \\
\hline $\mathrm{C} 14-\mathrm{OH}^{\mathrm{b}}$ & $0.02(0.01,0.04)$ & $0.05(0.04,0.06)$ & 5.49 & $<0.001$ \\
\hline
\end{tabular}

${ }^{a}$ data were normally distributed, and a $t$-test was used for comparison between the two groups; ${ }^{b}$ data did not conform to the normal distribution, and the rank-sum test was used for comparison between the two groups. PIH, pregnancy-induced hypertension; SD, standard deviation; M, median.
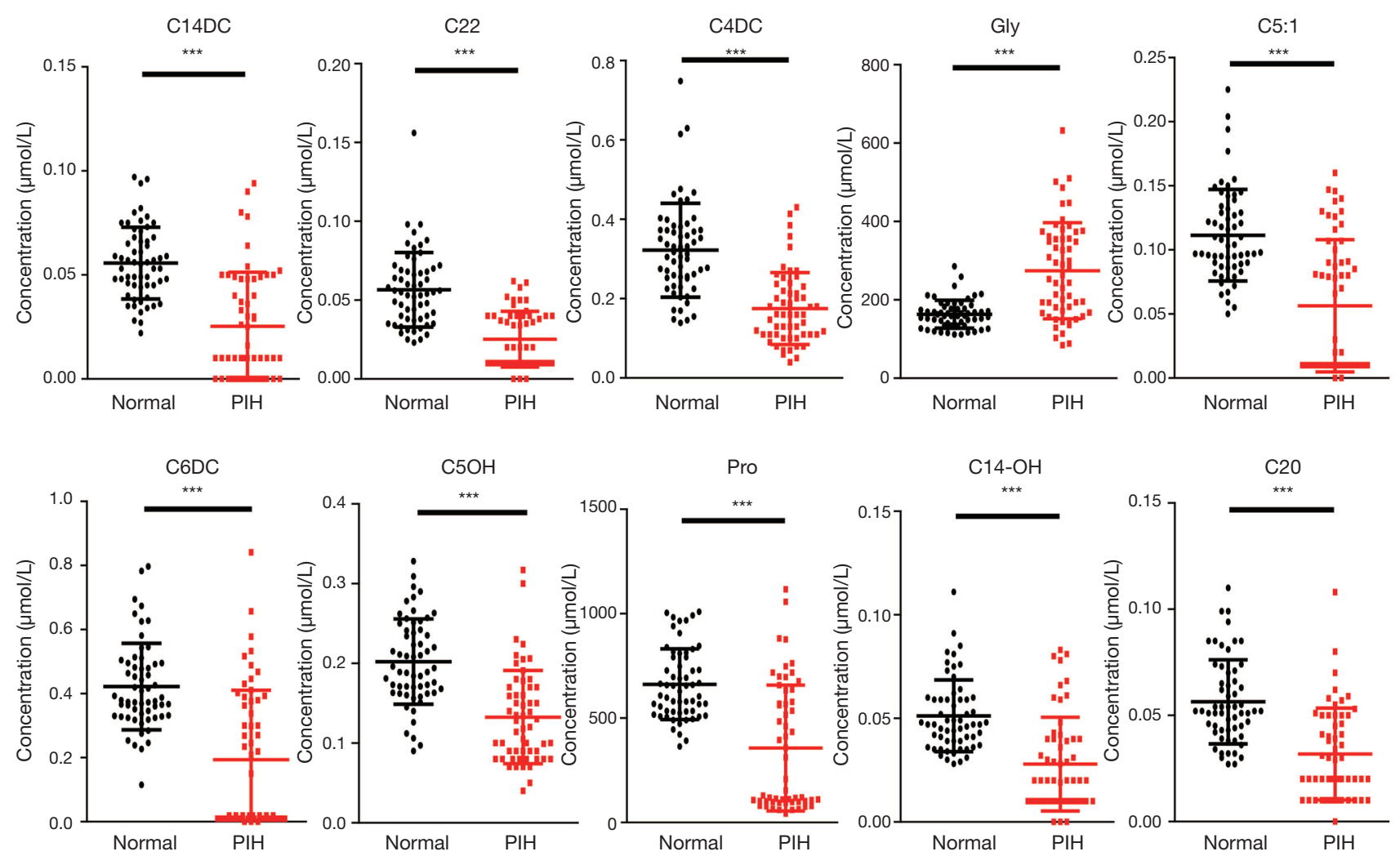

Figure 3 Scores plots for different metabolites in two groups. ${ }^{* * *} \mathrm{P}<0.001$. 


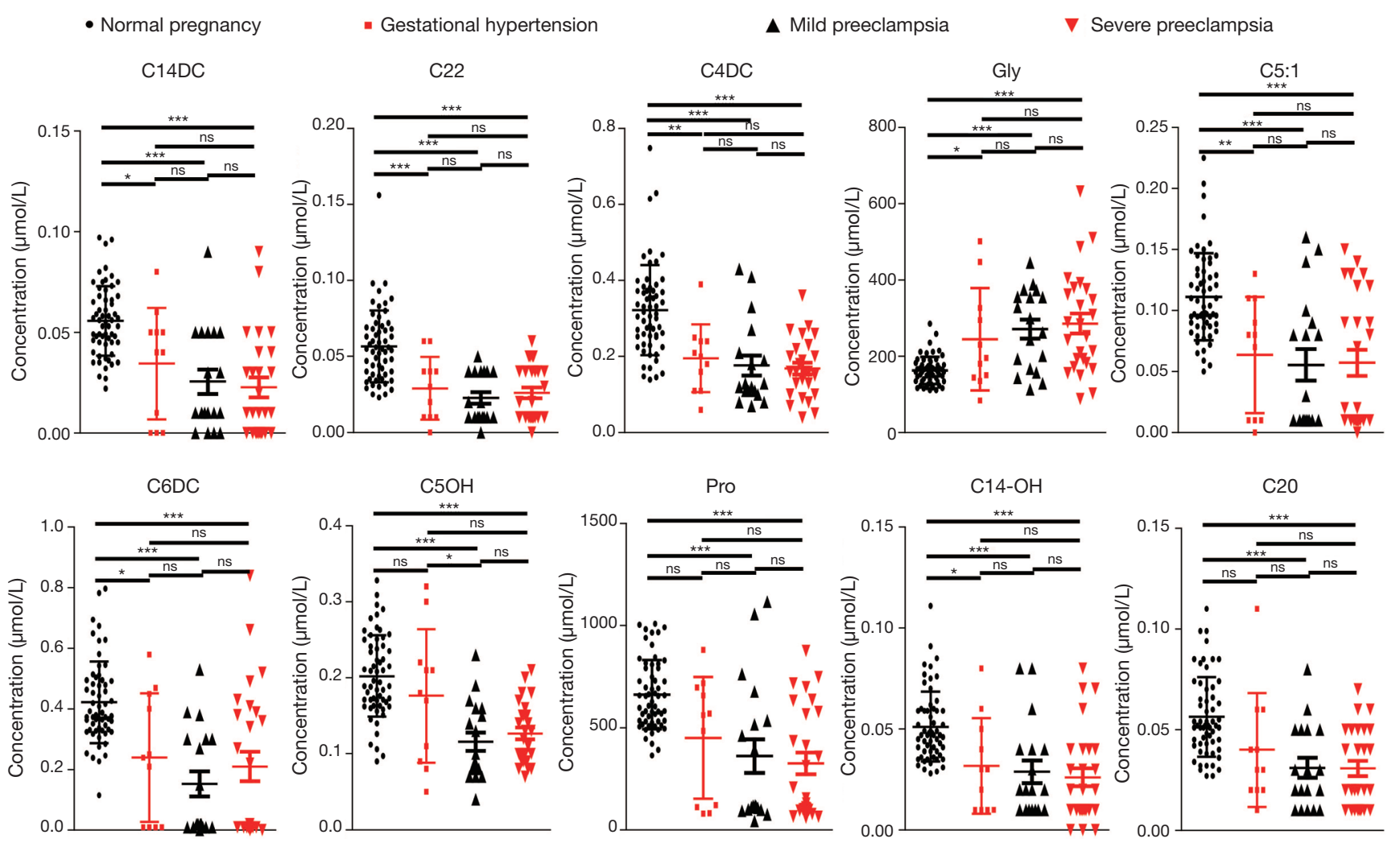

Figure 4 Scores plots for different metabolites in four groups. ${ }^{* *} \mathrm{P}<0.001 ;{ }^{* *} \mathrm{P}<0.01 ;{ }^{*} \mathrm{P}<0.05 ; \mathrm{ns}, \mathrm{P}>0.05$.

\section{Discussion}

In this study, based on the metabolomics platform, the blood metabolites of newborns of PIH mothers were analyzed by tandem mass spectrometry. The differences between these blood metabolites were studied to help evaluate the influence of gestational hypertension from mothers on newborns' blood metabolic status. All of the mothers were healthy except for PIH during their pregnancy. This study found a significant difference in blood metabolism between newborns with maternal PIH and those of mothers with normal pregnancy, which showed different metabolic characteristics.

Among 85 blood metabolites, 10 differential metabolites were found, which were different from those of normal pregnancy mothers. Compared with the normal pregnancy infant group, Gly showed an increasing trend in the neonatal group of mothers with PIH, while C14DC, C22, C4DC, C5:1, C6DC, C5-OH, Pro, C14-OH, and C20 showed a decreasing trend. This result was similar to the study of Wu et al. (20), who used tandem mass spectrometry to study the differences in blood metabolomes of preterm infants and term infants. They found 12 biomarkers such as free carnitine and succinyl carnitine, which decreased the preterm infant group. Li et al. (21) conducted a urine metabolomics study on very low weight births and found that stearic acid, palmitic acid, and myristic acid, among others, were significantly reduced in the very low birth weight group. At present, the side effects of PIH on newborns remain unclear. Studies have shown that the complication of hypertension in pregnancy accounted for $5 \%$ of all pregnancies, and was generally related to preterm labor (22). Our results also showed that PIH women are likely to experience preterm birth; there was a certain correlation between prematurely delivered fetuses and maternal PIH. Davies, Bell, and Bhattacharya (23) found that there was a positive correlation between PIH and premature delivery. Research by Omu, Othman, Al-Qattan, Al-Falah, and Sharma (24) also showed that compared with normal pregnant women, preterm birth incidence, caesarean birth, low birth weight, and perinatal mortality in women with PIH was significantly increased. Any of the 
symptoms caused by high blood pressure during pregnancy could be associated with many complications. Preterm birth is the main cause of perinatal morbidity, death, and longterm neurological sequelae. There are various complications such as bleeding, postpartum sepsis, bladder injury, and visceral trauma, which can be very serious, and low birth weight neonates are the leading division of neonatal death and morbidity. Saadat, Nejad, Habibi, and Sheikhvatan (25) considered that hypertension during pregnancy could cause complications such as respiratory distress, low birth weight, gestational diabetes, and maternal death. Wang et al. (26) showed that preeclampsia's clinical characteristics were similar in different hospitals in China, but the cesarean section rate was higher. Fallahian and Emahidosadati (27) found that pregnant women with gestational hypertension had a 7.5 -fold higher neonatal mortality rate than healthy pregnant women, and a premature birth rate that was 3.5 times that of healthy pregnant women. Odell et al. (28) recruited African women as participants to observe the impact of PIH on maternal and infant complications, and found that pregnant women with hypertension were a highrisk group for gestational diabetes, and $10 \%$ of these women gave birth to low-birthweight newborns. Considering the above discussion, this study's results may be applied to premature delivery, cesarean section, low birth weight, neonatal complications, and even death caused by PIH.

\section{Effects of amino acid changes on tissue and organ functions}

\section{Glycine}

Glycine is one of the non-essential amino acids and plays a very important physiological role in the anabolism of various substances in the body. Glycine is a precursor for various important metabolites such as glutathione, porphyrins, purines, haem, and creatine. These processes are mainly performed through the glycine cleavage system (GCS) and serine hydroxyl methyl transferase (SHMT) (29). Our study found that the increased glycine level of newborns in the PIH group may be related to the low GCS or SHMT enzyme activity in premature infants and low birth weight infants. What is more, glycine acts as a neurotransmitter in the central nervous system, and it has many roles such as antioxidant, anti-inflammatory, cryoprotective, and immunomodulatory in peripheral and nervous tissues. There were only 3 cases of neonatal asphyxia (3/56=5\%) in the PIH group, and all of them were mild asphyxia, which might be related to the compensatory protective mechanism of glycine in the body cells.

\section{Proline}

Proline is also one of the non-essential amino acids. Studies (30) have shown that proline content in the blood is positively correlated with newborns' birth weight, and the higher the content, the higher the incidence of macrosomia. Zhiqiang et al. (31) found that proline is the most critical biomarker in the process of interleukin (IL)-1-mediated chondrocyte metabolism, which can alleviate the metabolic disorder of osteoarthritis. Our study found that the decreased proline level was considered to be a risk factor for low birth weight of newborns with PIH mothers compared with those with normal maternal pregnancy. Moreover, we should avoid using drugs to affect the development of neonatal cartilage and monitor the newborn's cartilage development closely.

\section{Effects of fatty acid changes on tissue and organ functions}

Acyl carnitine is essential for intracellular energy metabolism, and characteristic changes in one or more acyl carnitines indicate the presence of a fatty acidoxidative metabolism disorder. Kido et al. (32) found that an increased level of acyl carnitine in the blood promoted fatty acids' metabolism, affected the metabolism of amino acids, and facilitated the production of energy of the heart and skeletal muscles. Scalais et al. (33) found that reduced levels of long-chain acyl carnitine led to cardiomyopathy, hepatic encephalopathy, severe hypoglycemia with low ketone, or sudden death. Makarova et al. (34) found that reduced levels of long-chain acyl carnitine were associated with insulin resistance in the muscles and heart. Our study detected decreased levels of different chain lengths of acyl carnitine in the PIH group, which showed us that the energy metabolism of the PIH group was weaker than in normal pregnancy, and that hypothermia, hypoglycemia, neonatal hypoxic ischemic encephalopathy, neonatal hyperbilirubinemia, and other complications are more likely to occur. It is important to provide sufficient energy during the early postnatal period to prevent the occurrence of fatty acid metabolic disorders in newborns of mothers with PIH.

Many current studies have shown that PIH could increase newborns' complications, but the mechanism is unclear (35). It has not been elucidated whether the neonatal outcome is more affected by the gestational age or the severity of hypertension during pregnancy. This study supports that PIH impacts neonatal metabolism, and screening of target metabolites can provide reasonable nutritional supplement recommendations for further correction of metabolic 
disorders.

\section{Acknowledgments}

Funding: This work was supported by funding from the Guangdong Provincial Science and Technology Plan Project, China (Z20150108201712159), the Science and Technology Foundation of Guangzhou, China (201704020230), the self-funded science and technology project of Foshan, China (2017AB001175), and the Natural Science Foundation of the Guangdong Province, China (2019A1515010637).

\section{Footnote}

Reporting Checklist: The authors have completed the STROBE reporting checklist. Available at http://dx.doi. org/10.21037/tp-20-211

Data Sharing Statement: Available at http://dx.doi. org/10.21037/tp-20-211

Peer Review File: Available at http://dx.doi.org/10.21037/tp20-211

Conflicts of Interest: All authors have completed the ICMJE uniform disclosure form (available at http://dx.doi. org/10.21037/tp-20-211). The authors have no conflicts of interest to declare.

Ethical Statement: The authors are accountable for all aspects of this work in ensuring that any questions related to the accuracy or integrity of any part of the work are appropriately investigated and resolved. The study was conducted in accordance with the Declaration of Helsinki (as revised in 2013). The study was approved by Ethics Committee of the Sixth Affiliated Hospital of Sun Yatsen University (2017ZSLYEC-105). All infant participants included in this study were recruited with the provision of written informed consent from their guardians.

Open Access Statement: This is an Open Access article distributed in accordance with the Creative Commons Attribution-NonCommercial-NoDerivs 4.0 International License (CC BY-NC-ND 4.0), which permits the noncommercial replication and distribution of the article with the strict proviso that no changes or edits are made and the original work is properly cited (including links to both the formal publication through the relevant DOI and the license). See: https://creativecommons.org/licenses/by-nc-nd/4.0/.

\section{References}

1. American College of Obstetricians and Gynecologists. ACOG practice bulletin no.202 gestational hypertension and preeclampsia. Obstet Gynecol 2019;133:e1-e25.

2. Xie Xin, Xun wenli. Obstetrics and gynecology (9th Ed.). Beijing: People's Medical Publishing House 2019.

3. Luo xiaolei, Wang tao. Key points of the 2019 ACOG clinical management guidelines for obstetricians and gynecologists with gestational hypertension. J Pract Obstet Gynecol 2019;35:259-262.

4. Gestational hypertension group, Chinese society of obstetrics and gynecology. Guidelines for diagnosis and treatment of gestational hypertension. Chinese Journal of Obstetrics and Gynecology 2015;50:721-8.

5. Regitz-Zagrosek V, Roos-Hesselink JW, Bauersachs J, et al. 2018 ESC guidelines for the management of cardiovascular diseases during pregnancy. Eur Heart J 2018;39:3165-241.

6. Berhe AK, Kassa GM, Fekadu GA. Prevalence of hypertensive disorders of pregnancy in Ethiopia: a systemic review and meta-analysis. BMC Pregnancy Childbirth 2018;18:34.

7. Carey RM, Whelton PK. Prevention, Detection, Evaluation, and Management of High Blood Pressure in Adults: Synopsis of the 2017 American College of Cardiology/American Heart Association Hypertension Guideline. Ann Intern Med 2018;168:351-8.

8. Gillon TE, Pels A, Dadelszen P, et al. Hypertensive Disorders of Pregnancy: A Systematic Review of International Clinical Practice Guidelines. PLoS One 2014;9:e113715.

9. Zhao S. Effects of hypertension during pregnancy on women's pregnancy outcomes. Chinese and Foreign Women's Health Research 2019;19:24+49.

10. Li Q. The harm of gestational hypertension. Health Science and Education 2019;40:78.

11. Clinical Mass Spectrometry Medical Committee, Laboratory Physician Branch, Chinese Medical Association. Application of MS/MS technology in screening of amino acid, organic acid and fatty acid oxidation and metabolism disorders in neonates. Laboratory Medicine 2019;34:479-85.

12. Bai J. Clinical application of tandem mass spectrometry in screening of genetic and metabolic diseases in neonates. Chinese Journal of Eugenics and Genetics 2008;26:67-9. 
13. Gika HG, Theodoridis GA, Plumb RS, et al. Current practice of liquid chromatography-mass spectrometry in metabolomics and metabonomics. J Pharm Biomed Anal 2014;87:12-25.

14. Zytkovicz TH, Fitzgerald EF, Marsden D, et al. Tandem mass spectrometric analysis for amino, organic, and fatty acid disorders in newborn dried blood spots: a two-year summary from the New England Newborn Screening Program. Clin Chem 2001;47:1945-55.

15. Zhou chunyan, Yao libo, et al. Biochemistry and molecular biology (9th Ed.). Beijing: People's Medical Publishing House, 2019.

16. Beale DJ, Pinu FR, Kouremenos KA, et al. Review of recent developments in GC-MS approaches to metabolomics-based research. Metabolomics 2018;14:152.

17. Zhou M, Jing JH, Mao RH, et al. Applications of metabonomics in animal genetics and breeding. Yi Chuan 2019;41:111-24.

18. Tang HR, Wang YL. Metabonomics: a Revolution in Progress. Progress in Biochemistry and Biophysics 2006;33:401-17.

19. Kelly RS, Giorgio R, Chawes B, et al. Applications of metabolomics in the study and management of preeclampsia: a review of the literature. Metabolomics 2017;13:86.

20. Wu Y, Wu S, Li S, et al. Application of tandem mass spectrometry to distinguish the markers of the blood metabolome of premature infants and full-term infants. Chinese Journal of Applied Clinical Pediatrics 2016;31:1325-8.

21. Li sitao, Huang xiaoling, Wu shiguang, et al. Study on urine metabolomics of very low birth weight premature infants. Chin J Pediatrics 2017;55:434-8.

22. Razak A, Florendo-Chin A, et al. Pregnancy-induced hypertension and neonatal outcomes: a systematic review and meta-analysis. J Perinatol 2018;38:46-53.

23. Davies EL, Bell JS, Bhattacharya S. Preeclampsia and preterm delivery: A population-based case-control study. Hypertension in Pregnancy 2016;35:510-9.

24. Omu AE, Al-Othman S, Al-Qattan F, et al. A comparative study of obstetric outcome of patients with pregnancy induced hypertension: economic considerations. Acta

Cite this article as: Hao H, Gu X, Cai Y, Xiong H, Huang L, Shen W, Ma F, Xiao X, Li S. The influence of pregnancyinduced hypertension syndrome on the metabolism of newborns. Transl Pediatr 2021;10(2):296-305. doi: 10.21037/tp-20211
Obstet Gynecol Scand 1996;75:443-8.

25. Saadat M, Nejad SM, Habibi G, et al. Maternal and neonatal outcomes in women with preeclampsia. Taiwanese Journal of Obstetrics and Gynecology 2007;46:255-9.

26. Wang V, Mueller A, Minhas R, et al. Understanding and comparing practices of managing patients with hypertensive disorders of pregnancy in urban China and the United States. Pregnancy Hypertens 2019;17:253-60.

27. Falahian M, Emahidosadati N. Effects of Pregnant Mothers' Pregnancy on Infants in Taleghani Hospital. Fertility Quarterly or Infertility 2000;2:48-53.

28. Odell CD, Kotelchuck M, Chetty V, et al. Maternal hypertension as a risk factor for low birth weight infants: Comparison of Haitian and African-American women. Matern Child Health J 2006;10:39-46.

29. Razak MA, Begum PS, Viswanath B, et al. Multifarious Beneficial Effect of Nonessential Amino Acid,Glycine: A Review. Oxid Med Cell Longev 2017;2017:1716701.

30. Koo WW, Krug W, Succop P, et al. Urinary hydroxyproline in infants with and without fractures/ rickets. Clin Chem 1990;36:642-4.

31. Wei Z, Dong C, Guan L, et al. A metabolic exploration of the protective effect of Ligusticum wallichii on IL-1 $\beta$-injured mouse chondrocytes. Chin Med 2020;15:12.

32. Kido J, Inoue H, Shimotsu H, et al. Effect of L-Carnitine on Amino Acid Metabolism in Elderly Patients Undergoing Regular Hemodialysis. Blood Purif 2020;49:614-21.

33. Scalais E, Bottu J, Wanders RJ, et al. Familial long chain acyl-CoA dehydrogenase deficiency as a cause of neonatal sudden infant death: improved survival by prompt diagnosis. Am J Med Genet A 2015;167A:211-4.

34. Makarova E, Makrecka-Kuka M, Vilks K, et al. Decreases in Circulating concentrations of Long-Chain acylcarnitines and Free Fatty Acids during the Glucose Tolerance Test represent Tissue-Specific Insulin sensitivity. Front Endocrinol (Lausanne) 2019;10:870.

35. Effect of gestational hypertension on fetal outcome, birth defects and related factors. Chinese Journal of Eugenics and Genetics 2018;26:107-10.

(English Language Editors: J. Jones and J. Chapnick) 